\title{
TIPOS POLÍNICOS DE LAS MIELES COMERCIALIZADAS DE GALICIA
}

\author{
María Carmen SEIJO COELLO \& María Jesús AIRA RODRÍGUEZ
}

\begin{abstract}
RESUMEN. Tipos polínicos de las mieles comercializadas de Galicia. Se ha realizado un estudio de las mieles de producción gallega existentes en el mercado. De las 39 muestras analizadas, tres han resultado monoflorales de Castanea, otras tantas monoflorales de Eucalyptus, cinco monoflorales de Rubus y el resto han sido milflorales. La mayoría de las mieles comercializadas proceden de las provincias de A Coruña y Lugo y en relación a sus características palinológicas, no difieren en gran medida de las procedentes de pequeños productores.
\end{abstract}

Palabras clave. Miel comercializada, polen, Galicia, España.

SUMMARY. Pollen types of commercial honey from Galicia. We have been realised a study of honeys produced in Galicia and distributed in the market. Of the 39 samples analysed, three have resulted uniflorals from Castanea, three uniflorals from Eucalyptus, five uniflorals from Rubus and the rest polyflorals. The main quantity of commercial honeys are from the provinces of A Coruña and Lugo. Related to their palynological characteristics, there are no important differences with honeys produced by small beekeepers.

Keywords. Commercial honey, pollen, Galicia, Spain

\section{INTRODUCCIÓN}

La producción de miel en Galicia tiene una gran tradición, ya antiguamente se utilizaban colmenas rústicas de tipo fijista llamadas trobos o cortizos, principalmente en zonas de montaña y zonas rurales, en donde se aseguraban cosechas otoñales de mieles, ricas en polen de castaño y brezo (Vieitez, 1951). A lo largo de los años, el sector apícola ha pasado por fases de importante desarrollo, al que ha contribuido la capacitación técnica de los apicultores (selección y renovación de reinas, tratamientos sanitarios, control de calidad del producto, etc.) y la utilización de colmenas movilistas.

En la actualidad, la miel y la cera, son los únicos productos de la colmena que se comercializan en Galicia, ya que el tipo de clima dificulta la obtención de polen, más factible en zonas más secas de España. Según los datos del Anuario de Estadística Agraria de 1997, en Galicia existen 118.000 colmenas con una producción total de miel de $1.500 \mathrm{Tm} / \mathrm{año}$.

En esta actividad agraria podemos diferenciar la apicultura llevada a cabo por pequeños productores, que por lo general poseen menos de 10 colmenas (mieles de autoconsumo) y la apicultura semiprofesional y profesional, con explotaciones de más de 30 
colmenas (mieles comercializadas) cuya producción puede llegar a mercados de mayor magnitud preferentemente a través de cooperativas.

Las mieles gallegas destinadas al autoconsumo y a la venta local, han sido objeto de numerosos trabajos en los últimos años (Aira et al., 1990, 1991; Iglesias et al., 1990, 1993; Janeiro et al., 1992; Jato \& Iglesias, 1989; Jato et al., 1991, 1992; Sánchez \& Sáenz, 1982; Seijo et al., 1992a,b,c y d, 1993, 1994a y b, 1995), mientras que las mieles comercializadas apenas han sido analizadas (Aira, 1990; Terradillos, 1984, 1988). Por ello, en el presente trabajo, se realiza un estudio de los tipos polínicos presentes en mieles accesibles al público en establecimientos comerciales con el fin de conocer cuales son las que caracterizan el mercado apícola gallego y la posibilidad de la existencia de fraudes en las mieles etiquetadas como producto gallego de calidad.

\section{MATERIAL Y MÉTODOS}

Se han analizado 39 muestras de miel comercializadas preferentemente a través de cooperativas y ampliamente distribuidas en establecimientos de alimentación de toda Galicia. A través de la dirección comercial de la etiqueta de envasado, se ha obtenido información de su lugar de procedencia y de la cantidad obtenida por cada productor (fig. 1, tab. 1). En algunos casos la miel procede de un solo colmenar, pero son frecuentes las mezclas de mieles de colmenares situados en distintas zonas y/o provincias. Todas las mieles han sido obtenidas por centrifugación, siendo las colmenas más utilizadas las de tipo movilista y de alzas.

Los métodos analíticos empleados han sido los propuestos por Louveaux et al. (1978). En todas las muestras se ha realizado el análisis cualitativo (contabilizándose un mínimo de

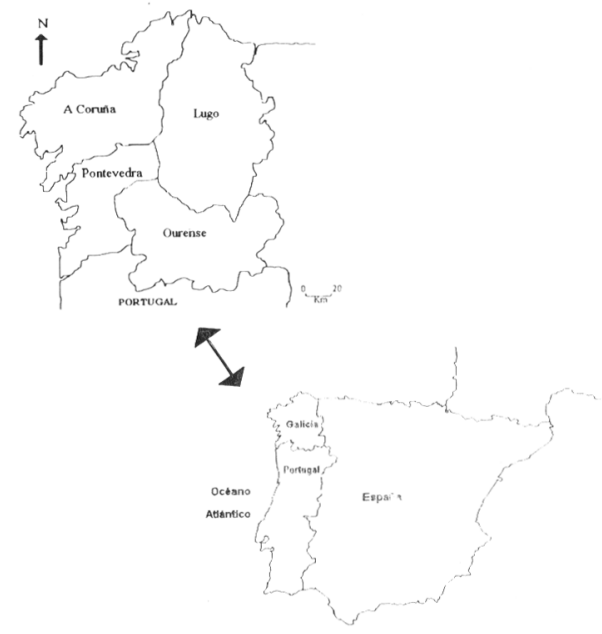

Figura 1. Situación geográfica del área de estudio. Geographical situation of the studied area.

1.200 granos de polen en cada preparación) y el cuantitativo (expresado en granos de polen contenidos en 10 gramos de miel) con el fin de conocer el origen floral, verificar su origen geográfico y su proceso de extracción.

Una vez obtenidos los resultados de los espectros polínicos se ha realizado un análisis de tipo cluster utilizando el paquete estadístico Statistica. que ha permitido organizar los espectros polínicos en dos grupos: mieles monoflorales (fig. 2) y mieles milflorales (fig. 3). A su vez este último grupo se ha podido subdividir en función de los resultados estadísticos derivados de sus características palinológicas en 4 grupos de mieles milflorales.

\section{RESULTADOS}

De las 39 muestras analizadas, once han resultado monoflorales (fig. 2) y 28 milflorales (fig. 3).

En las mieles monoflorales de castaño (un total de 3) se han incluido aquellas en las que el polen de Castanea está representado con 


\begin{tabular}{|c|c|c|c|}
\hline MIELES & LOCALIZACION & PRODUCCION* & RECOLECCION \\
\hline Castaño (1) & Lugo, Ourense & 30.000 & Julio/Agosto \\
\hline Castaño (2) & Ourense & 1.500 & Agosto \\
\hline Castaño (3) & Ourense & 2.000 & Julio/Agosto \\
\hline Eucalipto (4) & A Coruña & 1.750 & Septiembre \\
\hline Eucalipto (5) & A Coruña & 200 & Septiembre \\
\hline Eucalipto (6) & A Coruña & 1.500 & Julio/Agosto \\
\hline Zarza (7) & A Coruña & 400 & Septiembre/Octubre \\
\hline Zarza (8) & Lugo & 500 & Agosto/Octubre \\
\hline Zarza (9) & Lugo & 700 & Septiembre/Octubre \\
\hline Zarza (10) & Lugo & 1.500 & Septiembre \\
\hline Zarza (11) & Lugo & 350 & Septiembre \\
\hline Milfloral (12) & A Coruña & 400 & Septiembre \\
\hline Milfloral (13) & A Coruña & 300 & Septiembre \\
\hline Milfloral (14) & A Coruña, Lugo & 1.000 & Septiembre \\
\hline Milfloral (15) & A Coruña & 1.000 & Septiembre \\
\hline Milfloral (16) & A Coruña & 1.000 & Septiembre \\
\hline Milfloral (17) & A Coruña & 240 & Septiembre \\
\hline Milfloral (18) & Galicia & 15.000 & Julio/Septiembre \\
\hline Milfloral (19) & A Coruña & 300 & Septiembre \\
\hline Milfloral (20) & Galicia & 15.000 & Julio/Septiembre \\
\hline Milfloral (21) & Lugo & 1.000 & Septiembre \\
\hline Milfloral (22) & A Coruña & 1.800 & Agosto \\
\hline Milfloral (23) & A Coruña & 500 & Septiembre \\
\hline Milfloral (24) & Galicia & 80.000 & Julio/Septiembre \\
\hline Milfloral (25) & A Coruña & 375 & Septiembre \\
\hline Milfloral (26) & A Coruña & 1.000 & Septiembre \\
\hline Milfloral (27) & A Coruña & 3.000 & Agosto \\
\hline Milfloral (28) & Ourense & 10.000 & Septiembre \\
\hline Milfloral (29) & Lugo & 200 & Septiembre \\
\hline Milfloral (30) & Pontevedra & 900 & Julio/Agosto \\
\hline Milfloral (31) & A Coruña & 400 & Septiembre \\
\hline Milfloral (32) & Pontevedra & 700 & Septiembre \\
\hline Milfloral (33) & A Coruña & 500 & Octubre \\
\hline Milfloral (34) & Lugo & 250 & Septiembre \\
\hline Milfloral (35) & Ourense & 900 & Agosto \\
\hline Milfloral (36) & Lugo & 1.500 & Agosto/Octubre \\
\hline Milfloral (37) & Lugo & 6.000 & Septiembre \\
\hline Milfloral (38) & Lugo & 1.000 & Agosto/Septiembre \\
\hline Milfloral (39) & Lugo & 500 & Agosto/Septiembre \\
\hline
\end{tabular}

Tabla 1. Localización y producción de las mieles analizadas. * Kilogramos. Location and production of analysed honey. 


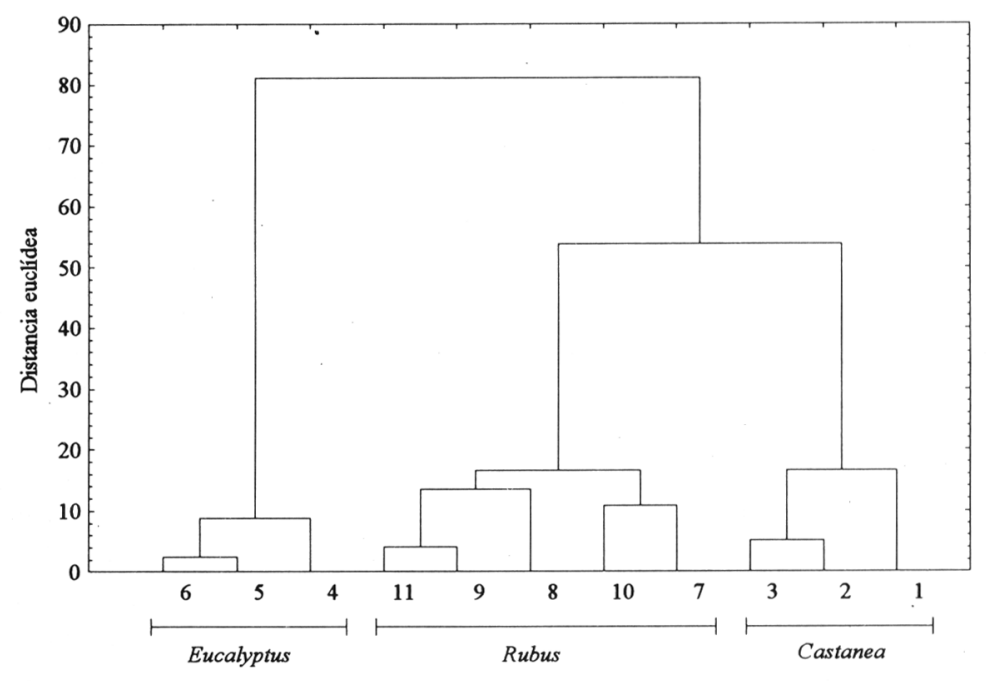

Figura 2. Dendrograma de agrupamiento de las mieles monoflorales. Cluster of monofloral honey.

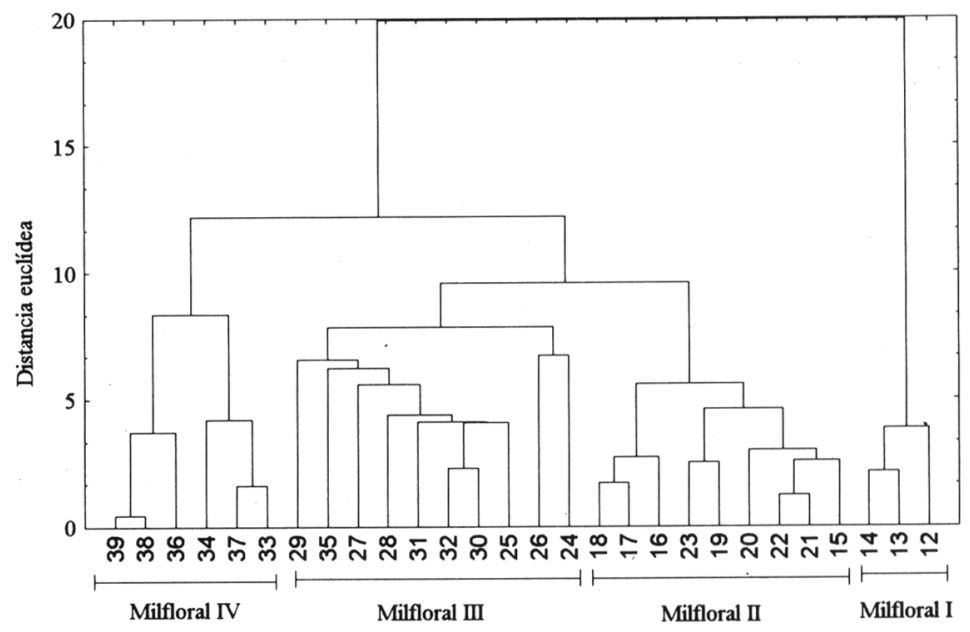

Figura 3. Dendrograma de agrupamiento de las mieles milflorales. Cluster of polyfloral honey. 
valores superiores al $73 \%$. En ellas, se han identificado 28 tipos polínicos, de los cuales Rubus es el más importante. La combinación Castanea-Rubus-Erica-Cytisus es común a todas las muestras.

En las mieles monoflorales de eucalipto (un total de 3) se incluyen aquellas mieles en las que el polen de Eucalyptus varía entre el $75 \%$ y el $85 \%$. En ellas se han identificado 31 tipos polínicos de los cuales Castanea-CytisusRubus están presentes en todas las muestras.

En las mieles monoflorales de zarza (un total de 5), el polen de dicho género aparece con porcentajes del $46,4 \%$ al $53,6 \%$. En estas mieles se han identificado 34 tipos polínicos, siendo la combinación de Rubus-CastaneaErica-Lotus la más representativa.

La distribución de los tipos polínicos de las mieles monoflorales en las cinco categorías propuestas por Louveaux et al. (1978), pone de manifiesto el predominio de los taxones incluidos en la categoría de «presentes» $(<1 \%$ del total del espectro polínico). Entre ellos se han identificado algunos exclusivamente poliníferos como es el caso de Plantago lanceolata y Poaceae cuya presencia en la miel es siempre escasa y que deriva de fuentes de contaminación secundaria del néctar.

El tipo polínico más frecuente en la categoría de «esporádicos» (1-3\%) en las mieles de castaño es Campanula, mientras que en las de eucalipto podemos citar a Rubus y en las de Rubus a Cytisus y Cistus psilosepalus. En la categoría de pólenes «importantes» (3$15 \%$ ) del espectro polínico de mieles de castaño destaca Rubus, en las de eucalipto Castanea y en las de Rubus el más frecuente es Lotus. En las mieles de castaño y eucalipto no se han identificado pólenes en la categoría de «acompañantes» (15-45\%) mientras que en las de Rubus se han encontrado Castanea y Erica.

Desde el punto de vista cuantitativo (fig. 4) las mieles monoflorales muestran una gran variabilidad incluyéndose en las Clases I, II, III y V de Maurizio. Paradójicamente dos mieles de Rubus han sido ser las de mayor y menor contenido en polen (miel 7 con 2.223.600 granos de polen y miel 10 con 6.950 granos de polen en 10 gramos de miel).

En las mieles milflorales están incluidas aquellas que no alcanzan los porcentajes mínimos estipulados para la denominación de monofloral. En estas mieles se han identificado 80 tipos polínicos diferentes y para su caracterización se han agrupado teniendo en cuenta su riqueza en polen de eucalipto (tab. 5).

En el grupo de Milflorales I (mieles 12, 13 y 14) se incluyen aquellas en las que el polen de Eucalyptus se encuentra representado con valores superiores al $60 \%$, también aparecen con porcentajes comprendidos entre el 15 y el $45 \%$ los tipos polínicos Castanea y Cytisus, mientras que los de Lotus, Sesamoides, Trifolium y Erica siempre presentan valores inferiores al $15 \%$.

En el grupo de Milflorales II (mieles 15 a 23) se incluyen las que presentan polen de eucalipto con valores comprendidos entre el 30-60\%. Estas mieles pueden presentar porcentajes elevados del polen del taxon Castanea sativa llegando incluso al $41 \%$ del total del espectro polínico. Además Rubus es con frecuencia polen importante junto a Erica, Cytisus y Lotus. Con valores inferiores al 3\% aparecen los pólenes de Brassica, Cistus psilosepalus, Salix, Trifolium y Echium.

En las Milflorales III (mieles 23 a 32 y $35)$ se incluyen aquellas cuyo contenido en polen de eucalipto varía entre 6-20\%. En el espectro polínico de nuevo aparecen con porcentajes elevados: Castanea cuyo máximo valor es de $68,9 \%$, junto a Rubus $(12,8 \%$ a $31,9 \%)$ y ocasionalmente Echium y Erica. Como pólenes importantes se han identificado Centaurea cyanus, Lotus, Trifolium, Cytisus y Hedera helix y como pólenes de menor importancia Quercus, Ligustrum, Scrophularia, Brassica y Salix.

En las Milflorales IV (mieles 33 a 39 


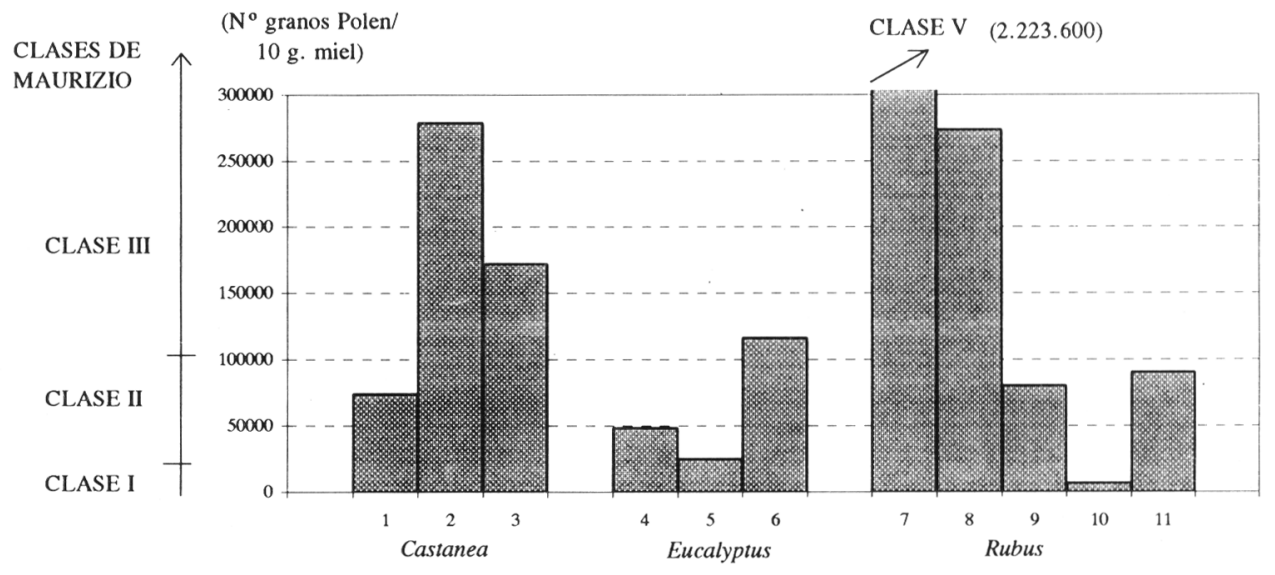

Figura 4. Número de granos de polen por 10 gramos de miel en cada una de las mieles monoflorales y su clase de Maurizio. Number of pollen grains per 10 grams of honey on each unifloral honey and their Maurizio's Class.

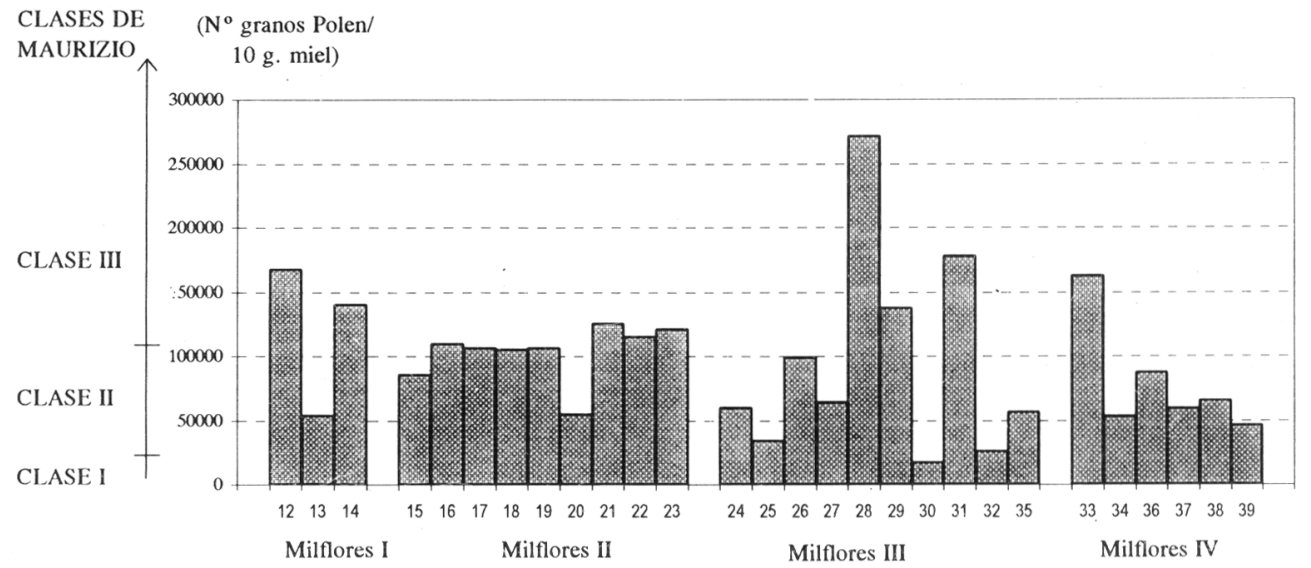

Figura 5. Número de granos de polen por 10 gramos de miel en cada una de las mieles milflorales y su clase de Maurizio. Number of pollen grains per 10 grams of honey on each polyfloral honey and their Maurizio's Class. 
excepto 35), el polen de eucalipto siempre presenta porcentajes inferiores al $6 \%$. El polen de Castanea es con frecuencia dominante siempre con valores superiores al $30 \%$, el polen de Rubus presenta valores en torno al $20 \%$ y ocasionalmente el tipo Cytisus es importante $(20,6 \%$ del polen total) y también se han encontrado, con valores inferiores al $15 \%$ los de Erica y Trifolium y con porcentajes menores del 3\% los pólenes de Centaurea cyanus, Campanula y Lotus.

En relación al contenido polínico (fig. 5), casi la totalidad de las mieles milflorales analizadas se incluyen en las Clases II y III de Maurizio. La miel 28 ha sido la de mayor riqueza polínica con 271.500 granos de polen / 10 gramos de miel (Clase III) y la miel 30 la más pobre con 17.650 granos de polen/10 gramos de miel (Clase I).

\section{DISCUSIÓN Y CONCLUSIONES}

Si comparamos las mieles comercializadas con las producidas preferentemente para autoconsumo (tab. 6), apenas se observan diferencias en los porcentajes de los taxones dominantes y tampoco en el contenido polínico, mientras que el número de tipos polínicos es ligeramente menor, lo cual puede ser debido a que la cantidad de mieles que llegan al mercado es más limitada reflejándose directamente en la media de tipos polínicos que presentan.

Desde el punto de vista cualitativo, las mieles comercializadas gallegas se limitan a mieles monoflorales de castaño, eucalipto y Rubus no habiéndose identificado otro tipo de mieles monoflorales que sí se encuentran entre las producidas para autoconsumo (Seijo et al., 1997). Los resultados obtenidos ponen de manifiesto el predominio de las mieles milflorales sobre las monoflorales y dentro de éstas, una mayor abundancia de las de Rubus, seguidas de las de castaño y eucalipto. Todas las mieles comercializadas que han sido analizadas, presentan espectros polínicos coherentes con la vegetación potencial del entorno donde han sido cosechadas, y por lo tanto su origen geográfico quedaría verificado con los análisis realizados.

En las mieles comercializadas de castaño, además del polen característico de este origen floral, es frecuente la presencia del polen de Rubus en cantidades elevadas y la mayoría contienen entre 100.000 a 500.000 granos de polen/10 gramos de miel. Las mieles comercializadas de eucalipto son ricas en polen de Castanea seguido de Cytisus y Rubus, y su contenido polínico es menor que las de castaño, oscilando en general, entre 20.000 y 100.000 granos de polen/10 gramos de miel. Las mieles comercializadas de Rubus presentan como pólenes acompañantes Castanea, Eucalyptus, Cytisus y Lotus, predominando las que se incluyen en la Clase II, al igual que ocurre con las de eucalipto.

Las mieles comercializadas de milflores se caracterizan por la presencia del polen de Castanea, Eucalyptus y Rubus, es decir de los taxones típicos de las mieles monoflorales. Estos taxones están frecuentemente acompañados por Cytisus, Erica, Trifolium, Campanula, etc. Por su contenido polínico se incluyen preferentemente en las Clases II y III, por lo tanto presentan entre 20.000 y 500.000 granos de polen/10 gramos de miel.

En cuanto a la distribución geográfica, las mieles de castaño proceden en su mayoría de las provincias de Lugo y Ourense y las de eucalipto y Rubus de A Coruña y Lugo. Las mieles milflorales proceden mayoritariamente de la provincia de A Coruña $(46,4 \%)$ y Lugo $(28,6 \%)$, mientras que en Ourense y Pontevedra, su producción es menor.

Como es de esperar, las mayores cantidades de miel proceden de aquellos apicultores que poseen sus colmenares en varias provincias. La provincia de A Coruña es la que aporta una mayor cantidad de miel al mercado seguida de Ourense y Lugo. La cantidad de 


\begin{tabular}{|c|c|c|c|c|c|c|c|c|c|c|c|c|c|c|c|}
\hline \multirow[b]{2}{*}{ Tipos polínicos } & \multicolumn{5}{|c|}{ Miel de Castanea } & \multicolumn{5}{|c|}{ Miel de Eucalyptus } & \multicolumn{5}{|c|}{ Miel de Rubus } \\
\hline & D & $\mathbf{A}$ & I & $\mathbf{R}$ & $\mathbf{P}$ & D & $\mathbf{A}$ & I & $\mathbf{R}$ & $\mathbf{P}$ & D & $\mathbf{A}$ & I & $\mathbf{R}$ & \\
\hline Anthyllis vulneraria & - & - & - & - & - & - & - & - & - & - & - & - & - & - & \\
\hline Apiaceae & - & - & - & - & - & - & - & - & - & 1 & - & - & - & - & \\
\hline Betula alba & - & - & - & - & - & - & - & - & - & 1 & - & - & - & - & \\
\hline Brassica & - & - & - & - & - & - & - & - & - & 2 & - & - & - & 1 & \\
\hline Calendula & - & - & - & - & 1 & - & - & - & - & - & - & - & - & - & \\
\hline Calluna vulgaris & - & - & - & - & - & - & - & - & - & 1 & - & - & 1 & 1 & \\
\hline Campanula & - & - & - & 2 & 1 & - & - & - & - & 2 & - & 3 & 1 & 1 & \\
\hline Castanea sativa & 3 & - & - & - & - & - & - & 3 & - & - & - & - & - & - & \\
\hline Centaurea cyanus & - & - & - & - & - & - & - & - & - & 1 & - & - & - & 2 & \\
\hline Cistus psilosepalus & - & - & - & - & 2 & - & - & - & - & 2 & - & - & - & - & \\
\hline Conium maculatum & - & - & - & - & 2 & - & - & 1 & - & - & - & - & - & - & \\
\hline Crataegus monogyna & - & - & - & - & 2 & - & - & - & - & 2 & - & - & - & 3 & \\
\hline Cytisus & - & - & 1 & 1 & 1 & - & - & 1 & 2 & - & - & - & 1 & - & \\
\hline Echium plantagineum & - & - & - & 1 & - & - & - & - & - & 1 & - & 1 & 1 & 1 & \\
\hline Erica & - & - & 1 & 1 & 1 & - & - & - & 1 & 2 & - & - & 2 & 2 & \\
\hline Eucalyptus globulus & - & - & - & - & 1 & 3 & - & - & - & - & - & - & - & - & \\
\hline Frangula alnus & - & - & - & - & 2 & - & - & - & - & - & - & - & - & - & \\
\hline Fraxinus & - & - & - & - & - & - & - & - & - & 1 & - & - & - & - & \\
\hline Galium & - & - & - & - & 3 & - & - & - & - & - & - & - & - & - & \\
\hline Hypericum & - & - & - & - & - & - & - & - & - & - & - & - & - & - & \\
\hline Ligustrum vulgare & - & - & - & - & - & - & - & - & - & 1 & - & - & - & - & \\
\hline Lithodora & - & - & - & - & 1 & - & - & - & - & - & - & - & - & - & \\
\hline Lotus & - & - & - & - & 2 & - & - & - & - & 2 & - & - & 3 & 2 & \\
\hline Mentha & - & - & - & - & 3 & - & - & - & - & - & - & - & - & - & \\
\hline Myosotis arvensis & - & - & - & - & 3 & - & - & - & - & 1 & - & - & - & - & \\
\hline Olea europaea & - & - & - & - & 1 & - & - & - & - & - & - & - & - & - & \\
\hline Papaver & - & - & - & - & - & - & - & - & - & - & - & - & - & & \\
\hline Pinus & - & - & - & - & - & - & - & - & - & - & - & - & - & & \\
\hline Plantago lanceolata & - & - & - & - & 2 & - & - & - & - & 2 & - & - & - & - & \\
\hline Poaceae & - & - & - & - & 1 & - & - & - & - & 2 & - & - & - & - & \\
\hline Polygonum persicaria & - & - & - & - & 1 & - & - & - & - & - & - & - & - & - & \\
\hline Prunus & - & - & - & - & - & - & - & - & - & 2 & - & - & - & & \\
\hline Quercus robur & - & - & - & - & 1 & - & - & - & 1 & 2 & - & - & - & & \\
\hline Ranunculus & - & - & - & - & - & - & - & - & - & 1 & - & - & - & & \\
\hline Reseda & - & - & - & - & - & - & - & - & - & 1 & - & - & - & & \\
\hline Resedaceae & - & - & - & - & - & - & - & - & - & 1 & - & - & - & & \\
\hline Rubus & - & - & 3 & - & - & - & - & - & 2 & 1 & 5 & - & - & & \\
\hline Rumex & - & - & - & - & - & - & - & - & - & - & - & - & - & & \\
\hline Salix & - & - & - & - & - & - & - & - & - & 2 & - & - & - & & . \\
\hline Scrophularia & - & - & - & - & - & - & - & - & - & 2 & - & - & - & & \\
\hline Sesamoides & - & - & - & - & 1 & - & - & - & - & 2 & - & - & - & & 1 \\
\hline Silene & - & - & - & - & 1 & - & - & - & - & - & - & - & - & & \\
\hline Sinapis & - & - & - & - & 1 & - & - & - & - & - & - & - & - & & \\
\hline Solanum & - & - & - & - & - & - & - & - & - & - & - & - & - & & \\
\hline Taraxacum officinale & - & - & - & - & 1 & - & - & - & - & - & - & - & - & & \\
\hline Teucrium scorodonia & - & - & - & - & 1 & - & - & - & - & - & - & - & - & & . \\
\hline Trifolium & - & - & - & 1 & - & - & - & - & 1 & 1 & - & - & 2 & & 2 \\
\hline Trifolium arvense & - & - & - & - & - & - & - & - & - & - & - & - & - & & - \\
\hline Zea mays & - & - & - & - & - & - & - & - & - & 2 & - & - & . & & \\
\hline
\end{tabular}

Tabla 2. Tipos polínicos de las mieles de castaño, eucalipto y Rubus.D (Polen dominante) $\geq 45 \%$; A (Polen acompañante) 15\%-45\%; I (Polen importante) 3\%-15\%; R (Polen esporádico) 1\%-3\%; P (Polen presente) $\leq 1 \%$. Pollen types of chestnut, Eucalyptus and Rubus honey. D (dominant pollen) $\geq 45 \%$; A (secondary pollen) $15 \%$ 45\%; I (important pollen) 3\%-15\%; $R$ (minor pollen) $1 \%-3 \% ; P$ (present pollen) $\leq 1 \%$. 


\begin{tabular}{lccccc} 
Tipos polínicos & D & A & I & R & P \\
\hline Castanea sativa & 8 & 14 & 6 & - & - \\
Eucalyptus globulus & 3 & 15 & 5 & 1 & 4 \\
Rubus & - & 6 & 20 & 2 & - \\
Cytisus & - & 3 & 17 & 3 & 1 \\
Erica & - & 2 & 11 & 7 & 7 \\
Lotus & - & 1 & 10 & 9 & 8 \\
Trifolium & - & 1 & 6 & 10 & 11 \\
Echium & - & 1 & - & 4 & 14 \\
Salix & - & - & 1 & 4 & 16 \\
Sesamoides & - & - & 1 & 4 & 11 \\
Cistus & - & - & 1 & 1 & 13 \\
Centaurea cyanus & - & - & 1 & 1 & 11 \\
Hedera helix & - & - & 1 & - & 4 \\
Calendula & - & - & 1 & - & - \\
Campanula & - & - & - & 7 & 18 \\
Brassica & - & - & - & 4 & 14 \\
Scrohpularia & - & - & - & 3 & 16 \\
Conium maculatum & - & - & - & 2 & 11 \\
Crataegus monogyna & - & - & - & 2 & 17 \\
Quercus robur & - & - & - & 2 & 20 \\
Ligustrum vulgare & - & - & - & 1 & 13 \\
Taraxacum oficcinale & - & - & - & 1 & 10 \\
Lythrum salicaria & - & - & - & 1 & 1 \\
Olea europaea & - & - & - & 1 & 1 \\
Anarrhinum & - & - & - & 1 & - \\
Rosmarinus officinalis & - & - & - & 1 & - \\
Plantago lanceolata & - & - & - & - & 21 \\
Poaceae & - & - & - & - & 21 \\
Prunus & - & - & - & - & 13 \\
Reseda & - & - & - & - & 11 \\
Lithodora & - & - & - & - & 9 \\
Mentha & - & - & - & - & 7 \\
Ranunculus & - & - & - & - & 7 \\
Teucrium scorodonia & - & - & - & - & 7 \\
Trifolium & - & - & - & - & 7 \\
Fraxinus & - & - & - & - & 6 \\
Rumex & - & - & - & - & 6 \\
Sedum & - & - & - & - & 6 \\
Pinus & - & - & - & - & 5 \\
Senecio & - & - & - & 5 \\
Anthemis & - & - & - & 4 \\
- & & & & &
\end{tabular}

\begin{tabular}{llllll} 
Tipos polínicos & D & A & I & R & P \\
\hline Myosotis & - & - & - & - & 4 \\
Papaver & - & - & - & - & 4 \\
Zea mays & - & - & - & - & 4 \\
Apiaceae & - & - & - & - & 3 \\
Frangula alnus & - & - & - & - & 3 \\
Ranunculaceae & - & - & - & - & 3 \\
Allium & - & - & - & - & 2 \\
Anthyllis & - & - & - & - & 2 \\
Arenaria & - & - & - & - & 2 \\
Aster & - & - & - & - & 2 \\
Betula alba & - & - & - & - & 2 \\
Hypericum perforatum & - & - & - & - & 2 \\
Ilex aquifolium & - & - & - & - & 2 \\
Malva & - & - & - & - & 2 \\
Silene vulgaris & - & - & - & - & 2 \\
Simethis planifolia & - & - & - & - & 2 \\
Solanum & - & - & - & - & 2 \\
Vitis vinifera & - & - & - & - & 2 \\
Acacia dealbata & - & - & - & - & 1 \\
Anagallis arvensis & - & - & - & - & 1 \\
Anthyllis vulneraria & - & - & - & - & 1 \\
Anchusa & - & - & - & - & 1 \\
Bellis & - & - & - & - & 1 \\
Raphanus & - & - & - & - & 1 \\
Bryonia cretica & - & - & - & - & 1 \\
Capsella & - & - & - & - & 1 \\
Cariophyllaceae & - & - & - & - & 1 \\
Cistus ladanifer & - & - & - & - & 1 \\
Citrus & - & - & - & - & 1 \\
Digitalis purpurea & - & - & - & - & 1 \\
Labiatae & - & - & - & - & 1 \\
Lavandula stoechas & - & - & - & - & 1 \\
Lonicera & - & - & - & - & 1 \\
Onobrychis & - & - & - & - & 1 \\
Papaveraceae & - & - & - & - & 1 \\
Resedaceae & - & - & - & - & 1 \\
Rosaceae & - & - & - & - & 1 \\
Serratula & - & - & - & - & 1 \\
Viola & - & - & - & - & 1 \\
Diola & & & & &
\end{tabular}

Tabla 3. Tipos polínicos de las mieles milflorales. D (Polen dominante) $\geq 45 \%$; A (Polen acompañante) 15\%-45\%; I (Polen importante) 3\%-15\%; R (Polen esporádico) 1\%-3\%; P (Polen presente) $\leq 1 \%$. Pollen types of polyfloral honey. D (dominant pollen) $\geq 45 \%$; A (secondary pollen) $15 \%-45 \%$; I (important pollen) $3 \%-15 \% ; R$ (minor pollen) $1 \%-3 \% ; P$ (present pollen) $\leq 1 \%$. 


\begin{tabular}{ccccccc}
\hline & \multicolumn{2}{c}{ \% Taxon dominante } & \multicolumn{2}{c}{$\mathbf{N}^{\circ}$ de tipos polínicos } & \multicolumn{2}{c}{ Clase de Maurizio } \\
\hline Miel & A & C & A & C & A & C \\
Monofloral de castaño & $70-93 \%$ & $73-92 \%$ & $8-35(\mathrm{X}=21)$ & $10-24(\mathrm{X}=17)$ & II, III, (IV) & II, III \\
Monofloral de Eucalipto & $70-93 \%$ & $76-85 \%$ & $28-24(\mathrm{X}=25)$ & $19-18(\mathrm{X}=18)$ & II, (I) & II, (III) \\
Monofloral de Zarza & $45-72 \%$ & $46-54 \%$ & $36-13(\mathrm{X}=24)$ & $12-18(\mathrm{X}=15)$ & II, (III) & II \\
Milflorales I & $>60 \%$ & $>60 \%$ & $14-35(\mathrm{X}=26)$ & $20-26(\mathrm{X}=23)$ & II, III & III, (II) \\
Milflorales II & $60-30 \%$ & $60-30 \%$ & $14-45(\mathrm{X}=29)$ & $19-38(\mathrm{X}=29)$ & II, I & II, III, (IV) \\
Milflorales III & $30-6 \%$ & $30-6 \%$ & $14-42(\mathrm{X}=28)$ & $11-45(\mathrm{X}=28)$ & II, (III) & III, (II) \\
Milflorales IV & $<6 \%$ & $<6 \%$ & $11-43(\mathrm{X}=17)$ & $11-24(\mathrm{X}=17)$ & II, III & II \\
\hline
\end{tabular}

Tabla 4. Características de las mieles de autoconsumo (A) y de las mieles comerciales (C). Characteristics of self-consumer honey $(A)$ and commercial honeys $(C)$.

miel procedente de la provincia de Pontevedra es comparativamente escasa, lo que en cierto modo justifica el bajo número de mieles monoflorales de eucalipto, ya que esta es la principal zona de producción de este tipo de mieles en Galicia (Seijo et al., 1998).

\section{BIBLIOGRAFÍA}

AIRA, M. J. -1990- Composición polínica y origen floral de seis mieles comerciales potencialmente gallegas. Rev. de Biología de la Universidad de Oviedo 8: 63-69.

AIRA, M. J., P. RAMIL y M. P. SAA -1990Identificación polínica de Ericaceae en mieles gallegas. Acta Bot. Malacitana 15: 27-32.

AIRA, M. J. IGLESIAS, V. JATO, A. SALALLINARES, M. C. SEIJO y M. SUAREZCER VERA - 1991- Mieles de Galicia (N. O. de España). Caracterización floral a través del espectro polínico. Historia Natural 91 Alemany A. (Ed), 109-114.

IGLESIAS, I., M. SUÁREZ-CERVERA, V. JATO y A. SALA-LLINARES -1990- Estudio de mieles de Castanea sativa Miller de la provincia de Orense (España). V Congreso Nacional de Apicultura. Don Benito, Badajoz.

IGLESIAS, I., V. JATO, y M. J. AIRA -1993Estudio palinológico de mieles de montaña de la provincia de Orense (N.O. España). Acta Bot. Malacitana 18:119-123.

JANEIRO, B., J. AMIGO y M.J. AIRA -1992Estudio secuencial de la miel de Paradapiñol (Quiroga, Lugo) y su relación con la flora melífera de la zona. Bol. de la Real Soc. Esp. de Hist. Nat. (Sección Biología), 88 (1-4): 213221.

JATO, V. e I. IGLESIAS - 1989- Oscilaciones del contenido polínico de mieles orensanas. Apiacta XXIV: $82-86$.

JATO, V., A. SALA-LLINARES, I. IGLESIAS \& M. SUAREZ-CERVERA -1991- Pollens of honeys from north-western Spain. Journal of Apicultural Research 30 (2): 69-73.

JATO, V., I. IGLESIAS y E. ALVAREZ -1992Variaciones interanuales del espectro polínico de mieles orensanas. Historia natural' 91. Alemany. A (Ed.), 115-122.

LOUVEAUX, J., A MAURIZIO \& G. VORWOHL -1978- Methods of melissopalynology. Bee World 59 (4): 139-157.

SÁNCHEZ, C. y C. SÁENZ -1982-Análisis polínico de mieles de Pontevedra. Lazaroa 4; 253-268.

SEIJO, M. C., M. J. AIRA y V. JATO -1992 aEstudio polínico de las mieles de la región Eurosiberiana gallega (N.O. España). IX Simposio de Palinología, Las Palmas.

SEIJO, M. C., M. J.AIRA y V. JATO -1992 bEvolución del espectro polínico de muestras de miel y néctar de un colmenar de Tomiño 
(Pontevedra). Bot. Complutensis 17: 87-97.

SEIJO, M. C., M. J. AIRA, I. IGLESIAS \& M. V. JATO - 1992 c- Palynological characterization of honeys from La Coruña province N.W. Spain). Journ. of Apicultural Research 31(3/4): 149-155.

SEIJO, M. C., V. JATO y M. J. AIRA -1992 dVariaciones intranuales del espectro polínico de la miel de Nogueira de Ramuín (Orense). Acta Bot. Malacitana 17: 175-182.

SEIJO, M. C., I. IGLESIAS, V. JATO y M. J. AIRA -1993- Oscilaciones polínicas de mieles de La Coruña (N.O. España). IV Simposio de Botánica, La Habana.

SEIJO, M. C., M. J. AIRA, I. IGLESIAS \& V. JATO -1994- L'activité d'affouragement de l'abeille domestique sur Actinidia deliciosa Chev. comprise la lumiere de l'analyse pollinique. Grana 33: 286-291.

SEIJO, M. C., V. JATO, M.J. AIRA \& I. IGLESIAS -1994- Caratterizazione pollinica dei mieli di Lugo (N.O. Spagna). Apicoltura 9: 7-18.

SEIJO, M. C., M. J. AIRA, I. IGLESIAS \& JATO, V. -1995-Origine botanique et zones de production des miels de Pontevedra (Nord-Ouest Espagne). Acta Bot. Gallica 142 (3): 235-243.

SEIJO, M. C., M. V. JATO, M. J. AIRA \& I. IGLESIAS - 1997- Uniflorals honeys of Galicia (North-West Spain). Journ. of Apicultural Research 36(3/4): 133-139

SEIJO, M. C., M. J. AIRA, y M. V. JATO -1998Distribución y características palinológicas de las mieles de Eucalyptus gallegas. Bot. Complutensis 22: 133-143.
TERRADILlOS, L. A. -1984- Contribución a la identificación de mieles monoflorales comerciales I. Algunos parámetros fisicoquimicos. II. Estudio microscópico del sedimento. Tesis de Licenciatura. Facultad de Farmacia. Universidad de Santiago, 78 p.

TERRADILLOS, L. A. - 1988-Estudio microscópico del sedimento de la miel y su aplicación en la caracterización de mieles de Galicia. Tesis doctoral. Facultad de Farmacia. Universidad de Santiago, 232 p.

VIEITEZ, E. -1951- El polen de las mieles de Galicia. Anales de Edafología y Fisiología Vegetal X: 79-99.

Aceptado para su publicación en Septiembre de 1999

Dirección de las autoras. M. C. SEIJO COELLO: Departamento de Biología Vegetal y Ciencia del Suelo. Facultad de Ciencias. Campus As Lagoas. Universidad de Vigo. 32004 Ourense. España. M. J. AIRA RODRÍGUEZ: Departamento de Biología Vegetal. Facultad de Farmacia. Campus Sur. 15706 Santiago de Compostela. España. 